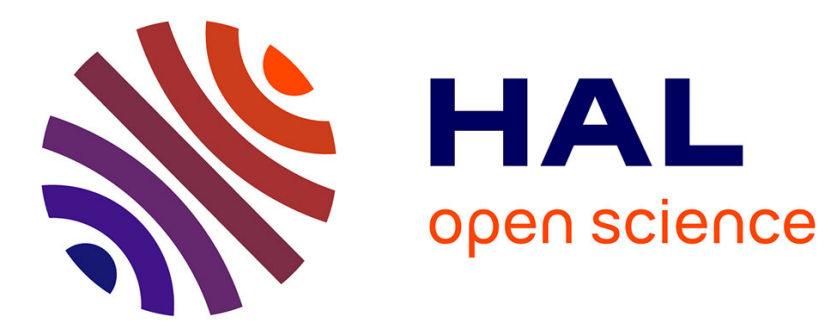

\title{
Glass-forming ability correlated with the liquid-liquid transition in Pd42.5Ni42.5P15 alloy
}

\author{
En-Yi Chen, Si-Xu Peng, Liang Peng, Marco Di Michiel, Gavin B.M. \\ Vanghan, Yao Yu, Hai-Bin Yu, Beatrice Ruta, Shuai Wei, Lin Liu
}

\section{- To cite this version:}

En-Yi Chen, Si-Xu Peng, Liang Peng, Marco Di Michiel, Gavin B.M. Vanghan, et al.. Glass-forming ability correlated with the liquid-liquid transition in Pd42.5Ni42.5P15 alloy. Scripta Materialia, 2021, 193, pp.117-121. 10.1016/j.scriptamat.2020.10.042 . hal-03093637

\section{HAL Id: hal-03093637 https://hal.science/hal-03093637}

Submitted on 26 Nov 2021

HAL is a multi-disciplinary open access archive for the deposit and dissemination of scientific research documents, whether they are published or not. The documents may come from teaching and research institutions in France or abroad, or from public or private research centers.
L'archive ouverte pluridisciplinaire $\mathbf{H A L}$, est destinée au dépôt et à la diffusion de documents scientifiques de niveau recherche, publiés ou non, émanant des établissements d'enseignement et de recherche français ou étrangers, des laboratoires publics ou privés. 
1 Glass-forming ability correlated with the liquid-liquid transition in

3 En-Yi Chen ${ }^{1,2}$, Si-Xu Peng ${ }^{2}$, Liang Peng ${ }^{1,2}$, Marco Di Michiel ${ }^{3}$, Gavin B. M. Vaughan ${ }^{3}$,

$5 \quad{ }^{1}$ State Key Laboratory of Materials Processing and Die \& Mould Technology, School of

6 Materials Science and Engineering, Huazhong University of Science and Technology,

7 Wuhan 430074, China.

$8{ }^{2}$ Wuhan National High Magnetic Field Center, Huazhong University of Science and

9 Technology, Wuhan 430074, China.

$10{ }^{3}$ ESRF — The European Synchrotron, CS40220, Grenoble 38043, France.

$11{ }^{4}$ Univ Lyon, Université Claude Bernard Lyon 1, CNRS, Institut Lumière Matière,

12 Villeurbanne, France.

13 5. Institute of Physics (IA), RWTH Aachen University, Aachen 52074, Germany.

$14 *$ Corresponding authors.

15 E-mail: ensiyu@mail.hust.edu.cn (Y.Y.); 1liu2000@mail.hust.edu.cn (L.L.)

\section{Abstract}

17 Alloy melts can solidify into metallic glasses if cooled fast enough to avoid crystallization.

18 Glass-forming ability (GFA), a measure of the ease of vitrification, is vital for the

19 fundamental understanding of glass formation, and is also crucial for the application of

20 metallic glasses. Previous studies of GFA mainly focused on the undercooled liquid phase,

21 while the influence of the evolution of the stable melts on GFA is rarely addressed. Here

22 we show that the $\mathrm{Pd}_{42.5} \mathrm{Ni}_{42.5} \mathrm{P}_{15}$ glass-forming liquid, in which a first-order liquid-liquid

23 transition (LLT) takes place at $T_{\mathrm{LL}}=1063 \mathrm{~K}$ high above its liquidus temperature, shows

24 significantly different GFA when quenched from the temperature above or below $T_{\mathrm{LL}}$.

25 Moreover, the pathway and kinetics of crystallization of the melt are strongly related to the 
26 kinetics of the LLT. Our work provides new insights into the vitrification process and the

27 kinetics of crystallization, and contributes to designing more stable metallic glasses.

\section{Keywords}

29 Crystallization, Phase transformation kinetics, Metallic glass, Time-temperature-

30 transformation, Nuclear magnetic resonance

31 
32 The unsatisfactory glass-forming ability is a major bottleneck of the structural 33 application of metallic glasses even though they show superior mechanical properties [1,

34 2]. Over the past half century, profound studies bring a series of breakthroughs in GFA of 35 metallic glass, which also deepen the fundamental understanding of the glass formation 36 and crystallization process [3-5]. As the formation of glass is a competing process between 37 supercooled liquid and crystalline phases, the understanding of GFA is mainly examined 38 through the lens of classical nucleation theory (CNT), where GFA is related to the crystal 39 nucleation rate per unit volume, $I_{\mathrm{n}}$, and low nucleation rate means good GFA [6-10]. Here $40 \quad I_{\mathrm{n}}$ is expressed as $I_{\mathrm{n}}=\frac{A_{\mathrm{v}}}{\eta(T)} \exp \left(-\frac{16 \pi \sigma^{3}}{3 k_{\mathrm{B}} T[\Delta g(T)]^{2}}\right)$, where $A_{\mathrm{v}}$ is a constant, $\eta(T)$ is the 41 viscosity of undercooled liquid, $\sigma$ is the interfacial energy between liquid and nuclei and $42 \Delta g(T)$ is the free energy difference between liquid and crystalline phases. Therefore, the 43 evolution of the supercooled liquid and the differences between the supercooled liquid and 44 crystalline phases are commonly believed to be the most important factors affecting GFA 45 [7, 11]. However, growing evidences of multistep nucleation with much higher nucleation 46 rate contradict the basic assumption of CNT, suggesting that the CNT encounters some 47 limitations in the interpretation of the kinetics and pathway of crystallization [12-16]. 48 These limitations strongly indicate that the current understandings of GFA are far from 49 comprehensive. Recently, more factors have been taken into consideration [17-20], while 50 there are still many issues about GFA which are not well understood.

51 Among all the factors that may affect GFA, the evolution of the alloy melt above its 52 liquidus temperature $T_{\text {liq }}$ is the least considered. The main reason is that the equilibrium 53 melt is commonly thought as a simple liquid with a very short $\left(\sim 10^{-13} \mathrm{~s}\right)$ relaxation time. 54 It seems impossible that the changes in the melt above $T_{\text {liq }}$ could affect the behaviors of 55 undercooled liquid, let alone the crystallization process [21]. Thus, it is commonly accepted 56 that the GFA is irrelevant to the state of equilibrium melt.

57 However, the situation could be quite different for the alloy melt with a liquid-liquid 58 transition taking place above $T_{\text {liq. }}$. Contrary to the conventional understanding, this melt is 59 very complex, and exhibits two distinct liquid phases [22]. In addition, the transition 60 between the two liquids shows the characteristics of first-order phase transition, which 
61 implies that both liquid states could be undercooled to the temperature far below $T_{\text {liq, }}$, even 62 approaching the glass transition temperature $T_{\mathrm{g}}$. Consequently, the influences of the 63 complexity of the alloy melts together with the kinetics of the first-order LLT should be 64 taken into consideration on the GFA of the melts.

65 Herein, a glass-forming alloy of $\mathrm{Pd}_{42.5} \mathrm{Ni}_{42.5} \mathrm{P}_{15}$ is selected, and the influence of LLT on 66 its GFA is studied. The liquidus temperature of the $\mathrm{Pd}_{42.5} \mathrm{Ni}_{42.5} \mathrm{P}_{15}$ system was determined 67 as $993 \mathrm{~K}$ by the flash differential scanning calorimetry (FDSC) (Fig. S1). In situ ${ }^{31} \mathrm{P}$ high68 temperature nuclear magnetic resonance (NMR) was carried out to characterize the LLT 69 above $T_{\text {liq. }}$. Firstly, step cooling experiments were conducted, in which the $\mathrm{Pd}_{42.5} \mathrm{Ni}_{42.5} \mathrm{P}_{15}$ 70 alloy was first heated to $1293 \mathrm{~K}$ and held there for $30 \mathrm{~min}$ for homogenization, and then it 71 was cooled step by step to $993 \mathrm{~K}$ with $40 \mathrm{~K}$ interval in the range of $1293 \sim 1093 \mathrm{~K}$ and 20 $72 \mathrm{~K}$ interval below $1053 \mathrm{~K}$. NMR spectra were taken isothermally after equilibrating for 30 $73 \mathrm{~min}$ at each step. The peak position of each spectrum, the Knight shift $\left(K_{\mathrm{s}}\right)$, which is 74 determined by the ensemble average of local magnetic field around ${ }^{31} \mathrm{P}$ nuclei and sensitive 75 to the changes in structure [22, 23], is plotted in Fig. 1a as a function of temperature $(T)$. $76 K_{\mathrm{s}}$ varies linearly above $1063 \mathrm{~K}$ with a slope of $-0.03 \mathrm{ppm} / \mathrm{K}$ (red line), but with a slope of $771.73 \mathrm{ppm} / \mathrm{K}$ (pink line) below $1063 \mathrm{~K}$, indicating a significant change in the P-centered 78 local structures. This result implies that an LLT may take place at $T_{\mathrm{LL}}=1063 \mathrm{~K}$. Two 79 typical ${ }^{31} \mathrm{P}$ spectra above and below $T_{\mathrm{LL}}$ are shown in the insets of Fig. 1a. Each spectrum 80 is a single Lorentzian peak and the integral areas of the peaks remain identical, indicating 81 that the system above or below $T_{\mathrm{LL}}$ is a homogeneous liquid without any loss of the 82 contributing $\mathrm{P}$ atoms.

83 In an ideal metallic liquid, the magnetic coupling between nuclei and $s$-like conduction 84 electrons through the Fermi contact interaction is the dominant mechanism of both Knight 85 shift and spin-lattice relaxation, leading to the Korringa relation $T T_{1} K_{\mathrm{s}}^{2}=\frac{\hbar \gamma_{\mathrm{e}}^{2}}{4 \pi k_{\mathrm{B}} \gamma_{\mathrm{n}}^{2}} f$, where $86 T_{1}$ is the spin-lattice relaxation time (Fig. S2), $\gamma_{\mathrm{e}}$ and $\gamma_{\mathrm{n}}$ are respectively the electron and 87 nucleus gyromagnetic ratio, $\hbar$ and $k_{\mathrm{B}}$ are respectively the reduced Planck and Boltzmann 88 constant, and $f$ is a constant related to the electron-electron interaction [24-26]. Thus, the 89 value of $T T_{1} K_{\mathrm{s}}^{2}$ is supposed to be a constant. However, in the presence of strong covalent 
90 bonds, the electrons tend to be localized, remaining in the vicinity of nuclei for longer time, 91 and thus the spin-lattice relaxation process would be more efficient. Therefore, the actual 92 relaxation time $T_{1}$ is shorter compared with the case in ideal metallic liquid, leading to the 93 breakdown of Korringa relation [27, 28]. Fig. $1 \mathrm{~b}$ plots the value of $T T_{1} K_{\mathrm{s}}^{2}$ versus $T$, which 94 is almost constant above $1063 \mathrm{~K}$, but drops substantially below $1063 \mathrm{~K}$ with decreasing 95 temperature, indicating the breakdown of Korringa relation. This result suggests that the 96 covalent bonding in the liquid is significantly enhanced below $1063 \mathrm{~K}$, which could be the 97 structural origination of the LLT.

98 Further, the kinetics associated with the LLT was examined. The sample was heated to $991173 \mathrm{~K}$ and held there isothermally for $30 \mathrm{~min}$, and then fast cooled to one of the 100 undercooled temperature (with respective to $T_{\mathrm{LL}}=1063 \mathrm{~K}$ ) $T_{\text {iso }}=1055,1053,1050,1047$, 1011045 , and $1043 \mathrm{~K}$ in about $1.5 \mathrm{~min}$, and held there isothermally. Once the temperature 102 stabilized at each $T_{\text {iso, }}$ NMR spectra were recorded every $10 \mathrm{~s}$ to monitor any possible 103 transition. Fig. 1c shows the measured $K_{\mathrm{s}}$ at different $T_{\text {iso. }}$. When reaching $T_{\text {iso }}, K_{\mathrm{s}}$ initially 104 follows the linear extrapolation (red line) of the $T$-dependent $K_{\mathrm{S}}$ above $1063 \mathrm{~K}$ measured 105 in the step cooling experiments. After a certain incubation time $\tau, K_{\mathrm{s}}$ starts to jump to the 106 expected value determined by the linear fit of the $T$-dependent $K_{\mathrm{s}}$ below $1063 \mathrm{~K}$ (pink line). 107 These results clearly show that the stable liquid above $1063 \mathrm{~K}$ (high-temperature liquid, 108 named as HTL) can be undercooled below $T_{\mathrm{LL}}$ and finally transforms to the stable liquid 109 below $1063 \mathrm{~K}$ (low-temperature liquid, named as LTL). Such behavior is consistent with 110 the first-order phase transition [29]. The average incubation time $\tau$ of three independent 111 measurements at each temperature versus the degree of undercooling $\Delta T=T_{\mathrm{LL}}-T_{\text {iso }}$ is 112 shown in Fig. 1d. As $\Delta T$ increases from 8 to $20 \mathrm{~K}$ (i.e., $T_{\text {iso }}$ decreases from 1055 to 1043 $113 \mathrm{~K}$ ), the incubation time decreases rapidly from $240 \mathrm{~s}$ to $30 \mathrm{~s}$. The inset shows the plot of $114 \log \left(T_{\text {iso }} / \tau\right)$ versus $\log (\Delta T)$, which can be well fitted with a linear function, indicating a 115 power-law relation of $\tau \propto T_{\text {iso }} /(\Delta T)^{m}$ with $m=2.1$. Similar power-law was also observed 116 in the LLTs in $\mathrm{La}_{50} \mathrm{Al}_{35} \mathrm{Ni}_{15}$ liquid [22] and molten sodium acetate trihydrate [30].

117 To further explore the difference between HTL and LTL, two glassy samples, labeled as 118 HTA and LTA accordingly, were prepared by quenching the melt from $1223 \mathrm{~K}$ (above $T_{\mathrm{LL}}$ ) 
119 and $1023 \mathrm{~K}$ (below $T_{\mathrm{LL}}$ ) using melt-spinning technique, respectively. High-energy 120 synchrotron X-ray diffraction (XRD) was then employed to examine the structure of the 121 two glassy samples. As shown in Fig. 2a, no sharp Bragg peaks corresponding to any 122 crystallites are detected on the structure factors $S(q)$, confirming the amorphous nature of 123 the two samples. These results are consistent with the high-resolution transmission electron 124 microscopy (HRTEM) results (Fig. S3). The full width at half maximum (FWHM) of the 125 first peak in $S(q)$ are about $0.44 \AA^{-1}$ and $0.34 \AA^{-1}$ for HTA and LTA, respectively. The 126 narrower FWHM of LTA suggests the existence of more ordered structures in this glass.

127 The structural details can be better appreciated from real space analysis by looking at the 128 pair correlation function $G(r)$ obtained by the Fourier transformation of the corresponding $129 S(q)$ (Fig. 2b). The positions difference of the first maxima of $G(r)$ is very subtle (2.75 130 for LTA and $2.74 \AA$ for HTA). The increased order in the structure of the LTA is reflected 131 in well-defined oscillations up to the $12^{\text {th }}$ shell in the LTA while only up to the $8^{\text {th }}$ shell in 132 the HTA. This behavior is clearly identified from the difference in $G(r)$ between HTA and 133 LTA, obtained by subtracting the former from the latter (inset of Fig. 2b). The difference 134 between these two $G(r)$ curves are negligible up to the second minimum distance $(r<5.9$ $135 \AA$ ), but beyond this distance, LTA shows an enhanced correlation which is still pronounced 136 even up to $r>25 \AA$. These results suggest that the structure of short-range clusters in LTA 137 and HTA are almost the same, but a more pronounced medium-range ordering presents in 138 LTA.

139 LLTs were supposed to take place in several metallic glass-forming liquids, but the 140 structural mechanism associated with the LLT was not clear [31-33]. NMR observables are 141 sensitive to the interaction between electrons and nuclei, and can provide information of 142 all nearest-neighbor atoms surrounding the atom and possibly beyond. Combined with the 143 evidence of synchrotron XRD, more detailed structural changes can be revealed. Since the 144 glass is commonly considered as "frozen liquid", the results of HTA and LTA imply that 145 the structures of short-range clusters in LTL and HTL could be almost identical, but the 146 correlation between the short-range clusters in LTL and HTL should be different. Recalling 147 the significantly enhanced covalent bonding in LTL suggested by the breakdown of 148 Korringa relation (Fig. 1b), it is reasonable to assume that the transition from HTL to LTL 
149 is associated with the increase of the short-range clusters and stronger correlation between

150 the clusters, consistent with the two-order-parameter model [34, 35].

151 The GFA of the two liquids were examined by FDSC experiments through measuring 152 the critical cooling rate $R_{\mathrm{c}}$, which is the slowest cooling rate at which equilibrium liquid 153 can be quenched into glass without detectable crystallites, and a lower $R_{\mathrm{c}}$ stands for a better 154 GFA [36, 37]. The sample was first heated to a predetermined temperature $T_{\mathrm{q}}=1073$ (i.e., $15510 \mathrm{~K}$ above $T_{\mathrm{LL}}$ ) at a constant rate of $100 \mathrm{~K} / \mathrm{s}$ and kept there isothermally for $30 \mathrm{~s}$, then 156 cooled to room temperature at a series of cooling rate $q^{-}(50 \sim 40000 \mathrm{~K} / \mathrm{s})$ to obtain samples 157 with different content of amorphous phase. After this pretreatment, the contents of 158 amorphous phase in these samples were measured by FDSC via heating each sample from 159 room temperature to $1073 \mathrm{~K}$ at a constant rate of $100 \mathrm{~K} / \mathrm{s}$ (see the inset of Fig. 3a for 160 temperature protocol). For comparison, these procedures were repeated for $T_{\mathrm{q}}=1023 \mathrm{~K}$ 161 (i.e., $40 \mathrm{~K}$ below $T_{\mathrm{LL}}$ ). Fig. 3a shows a representative DSC heating curve for the sample 162 obtained from $T_{\mathrm{q}}=1073 \mathrm{~K}$ and $q^{-}=40000 \mathrm{~K} / \mathrm{s}$. The two exothermic peaks (around $685 \mathrm{~K}$ 163 and $770 \mathrm{~K}$ ) are related to the polyamorphic transition [38,39] and the crystallization of the 164 amorphous phase, respectively, and the endothermic peaks (around $880 \mathrm{~K}$ ) represent the 165 melting process. Fig. $3 \mathrm{~b}$ plots the area of the second exothermic peak $(\Delta H)$ versus $T$. Here, $166 \Delta H$ of each sample was normalized to that of the sample obtained from $T_{\mathrm{q}}=1073 \mathrm{~K}$ and $167 q^{-}=40000 \mathrm{~K} / \mathrm{s}$ (this sample was used as the reference because it is fully amorphous, see 168 the discussion below), and this value is directly proportional to the content of amorphous 169 phase in each sample. For samples cooled from $T_{\mathrm{q}}=1023 \mathrm{~K}$ (i.e., from LTL), the content 170 of amorphous phase does not change when $q^{-}>6000 \mathrm{~K} / \mathrm{s}$, but drops significantly when $q^{-}$ $171<6000 \mathrm{~K} / \mathrm{s}$, suggesting that $R_{\mathrm{c}}$ is about $6000 \mathrm{~K} / \mathrm{s}$ for LTL. However, for samples cooled 172 from $T_{\mathrm{q}}=1073 \mathrm{~K}$ (i.e., from HTL), the content of amorphous phase remains constant until $173 q^{-}<100 \mathrm{~K} / \mathrm{s}$, indicating that $R_{\mathrm{c}}$ is about $100 \mathrm{~K} / \mathrm{s}$ for HTL. The results demonstrate clearly 174 that HTL exhibits much better GFA compared to LTL.

175 The significance of the current experiments is that the GFA is observed to be closely 176 related to the liquid-liquid transition event. It is shown unambiguously that an alloy melt 177 can exhibit two significantly different GFA without change in composition. Due to the 178 first-order nature of the LLT, both HTL and LTL can be maintained to the temperature far 
179 below $T_{\text {liq, }}$, and further vitrified into different glasses (Fig. $2 \mathrm{a}$ ). The evolutions of the two 180 liquids are schematically shown in Fig. 4a. As HTL and LTL are distinct liquid states, it is 181 reasonable that they show different crystallization kinetics and hence different GFAs.

182 The LLT plays a crucial role in the crystallization of HTL. On the one hand, it provides 183 an alternative crystallization pathway for HTL. The crystallization of HTL would follow 184 one of the two possible pathways: either crystallize directly from HTL state (Path I in Fig. 185 4a), or crystallize via LTL after the LLT takes place (Path II in Fig. 4a). In the latter case, 186 the supercooled LTL acts as an intermediate state between the supercooled HTL and 187 crystalline state. The existence of Path II is coincident with previous studies [38, 39], where 188 it was suggested that the HTA transformed firstly to a supercooled liquid (supercooled 189 liquid I) above its glass transition temperature, and then to another supercooled liquid 190 (supercooled liquid II) before crystallization upon further heating. Although the 191 supercooled liquid I and II are not cognized in detail, they are consistent with the 192 supercooled HTL and LTL in our picture shown in Fig. 4a, respectively. Thus, our 193 experiments suggest a new possible crystallization pathway upon cooling, i.e., crystallize 194 via an intermediate liquid state (Path II).

195 On the other hand, the LLT may also change the crystallization kinetics of HTL. The 196 selection of the crystallization pathway is determined by the competition between the 197 kinetics of LLT and the crystallization kinetics of HTL. Once the incubation time of LLT 198 is shorter than that of the crystallization of HTL, HTL would crystallize through Path II. 199 Fig. 4b schematically shows a possible relationship between the crystallization time200 temperature-transformation (TTT) curves of HTL (green dashed line) and LTL (red line), 201 together with the TTT curve of LLT (cerulean line). Here, the critical cooling rate of HTL, $202 R_{\mathrm{c}}^{\mathrm{HTL}}$, is no longer related to the intrinsic TTT curve of HTL, but related to the effective203 TTT curve (dark dotted line), which is the superposition of the TTT curve of LLT and the 204 crystallization TTT curve of LTL, with its nose point around the lower intersection of the 205 two curves. The minimum cooling rate to bypass this effective-TTT curve can be 206 determined as $R_{\mathrm{c}}=\left(T_{\mathrm{LL}}-T_{\mathrm{en}}^{\mathrm{HTL}}\right) / t_{\mathrm{en}}^{\mathrm{HL}}$, where $T_{\mathrm{en}}^{\mathrm{HTL}}$ and $t_{\mathrm{en}}^{\mathrm{HTL}}$ are the temperature and time 207 scale at the nose point. By assuming that $T_{\mathrm{en}}^{\mathrm{HTL}}$ is around the nose temperature $T_{\mathrm{n}}^{\mathrm{LTL}}$ of the 
208 TTT curve of LTL, which can be estimated as $T_{\mathrm{n}}^{\mathrm{LTL}}=T_{\mathrm{g}}+0.5\left(T_{\text {liq }}-T_{\mathrm{g}}\right)=780 \mathrm{~K}$ [40] and $209 t_{\mathrm{en}}^{\mathrm{HTL}}$ deduced from the continuous-cooling-transformation (CCT) curve of LLT 210 constructed by the method developed by Grange and Keifer [41], is about 1.4 s (Fig. S4), $211 R_{\mathrm{c}}$ can be roughly estimated as $200 \mathrm{~K} / \mathrm{s}$. To our great surprise, this value is very close to 212 the $R_{\mathrm{c}}^{\mathrm{HTL}}$ acquired from the FDSC experiments. This result strongly indicates that the 213 picture described in Fig. $4 b$ could be the case of the crystallization in $\mathrm{Pd}_{42.5} \mathrm{Ni}_{42.5} \mathrm{P}_{15}$.

214 It is of interest to note that HTL exhibits a better GFA than LTL. Although the detailed 215 kinetics of the direct crystallization process of HTL is experimentally unachievable due to 216 the interference of LLT, it can be concluded qualitatively from the poor GFA of LTL that 217 the crystal nucleation rate per unit volume $I_{\mathrm{n}}$ from LTL should be much higher than that 218 from HTL. This may result from the formation of the clusters with medium-range ordering 219 in the supercooled LTL, and such clusters may act as precursors for the nucleation of the 220 crystalline phase [11], reducing the interfacial energy $\sigma$ and leading to a lower energy 221 barrier for the formation of the crystal nuclei and hence the poor GFA of LTL.

222 In summary, our in situ high-temperature NMR studies show that the glass-forming 223 liquid $\mathrm{Pd}_{42.5} \mathrm{Ni}_{42.5} \mathrm{P}_{15}$ presents a first-order LLT at the temperature of $1063 \mathrm{~K}$, high above 224 the liquidus temperature of $993 \mathrm{~K}$, accompanied by a drastic enhancement of the covalent 225 bonding below $1063 \mathrm{~K}$. The two liquids can be vitrified into two corresponding glasses 226 which are different in the medium-range ordering rather than the structure of short-range 227 clusters. These results suggest that the LLT may be a result of the increase of the short228 range clusters. The FDSC studies reveal that the high-temperature liquid exhibits much 229 better GFA than the low-temperature liquid, which may be ascribed to the enhancement of 230 medium-range ordering in the supercooled LTL. Further analyses indicate that the LLT can 231 provide an indirect crystallization pathway for HTL, and moreover, the kinetics of LLT 232 has a significant influence on the GFA. This effects of LLT provides a new way for future 233 study of the process of crystallization and is also important for the fabrication of metallic 234 glasses. What is more, our work may also provide an alternative understanding for some 235 traditional phenomena. For instance, many metallic glass-forming liquids exhibit 236 overheating effects, where the nucleation behavior of the system or the mechanical 237 properties of the metallic glasses show markedly difference with different overheating 
temperature $[42,43]$. Till now, there are only qualitative understandings for these

239 phenomena. It is proposed to be related to the heterogeneities in the liquid, which dissolve

240 above a threshold temperature. However, such behavior could be quantitatively explained

241 within our LLT scenario, where $T_{\mathrm{LL}}$ is the threshold temperature.

242

243

244 Declaration of Competing Interest

245 The authors declare that they have no known competing financial interests or personal

246 relationships that could have appeared to influence the work reported in this paper.

247 Acknowledgement

248 The authors are grateful to the Analytical and Testing Center of HUST for technical 249 assistance. This work was financially supported by the National Natural Science 250 Foundation of China (Grant No. 51872105).

\section{References}

252 [1] W.H. Wang, C. Dong, C.H. Shek, Mater. Sci. Eng. R Rep. 44(2) (2004) 45-89.

253 [2] M. Telford, Mater. Today 7(3) (2004) 36-43.

254 [3] A.L. Greer, Science 267(5206) (1995) 1947.

255 [4] Y. Li, Q. Guo, J.A. Kalb, C.V. Thompson, Science 322(5909) (2008) 1816.

256 [5] C.G. Tang, P. Harrowell, Nat. Mater. 12(6) (2013) 507-511.

257 [6] M.H. Cohen, D. Turnbull, J. Chem. Phys. 31(5) (1959) 1164-1169.

258 [7] D. Turnbull, Contemp. Phys. 10(5) (1969) 473-488.

259 [8] A. Inoue, Acta Mater. 48(1) (2000) 279-306.

260 [9] Z.P. Lu, C.T. Liu, Acta Mater. 50(13) (2002) 3501-3512.

261 [10] D.M. Herlach, Mater. Sci. Eng. R Rep. 12(4) (1994) 177-272.

262 [11] J. Russo, F. Romano, H. Tanaka, Phys. Rev. X 8(2) (2018) 021040.

263 [12] D. Erdemir, A.Y. Lee, A.S. Myerson, Acc. Chem. Res. 42(5) (2009) 621-629.

264 [13] P.G. Vekilov, Cryst. Growth Des. 10(12) (2010) 5007-5019.

265 [14] J. De Yoreo, Nat. Mater. 12(4) (2013) 284-285.

266 [15] J. Lee, J. Yang, S.G. Kwon, T. Hyeon, Nat. Rev. Mater. 1(8) (2016) 16034.

267 [16] J.J. De Yoreo, P.U.P.A. Gilbert, N.A.J.M. Sommerdijk, et al., Science 349(6247) 268 (2015) aaa6760.

269 [17] P. Zalden, F. Quirin, M. Schumacher, et al., Science 364(6445) (2019) 1062.

270 [18] S. Wei, G.J. Coleman, P. Lucas, C.A. Angell, Phys. Rev. Appl 7(3) (2017) 034035.

271 [19] J. Schroers, W.L. Johnson, J. Appl. Phys. 88(1) (2000) 44-48. 
272 [20] J. Schroers, Y. Wu, R. Busch, W.L. Johnson, Acta Mater. 49(14) (2001) 2773-2781.

273 [21] A. Cavagna, Phys. Rep. 476(4) (2009) 51-124.

274 [22] W. Xu, M.T. Sandor, Y. Yu, et al., Nat. Commun. 6 (2015) 7696.

275 [23] L.L. Li, J. Schroers, Y. Wu, Phys. Rev. Lett. 91(26) (2003) 265502.

276 [24] A. Abragam, Principles of Nuclear Magnetism, Clarendon Press, Oxford, 1961.

277 [25] C.P. Slichter, Principles of Magnetic Resonance, Harper \& Row Publishers, New 278 York, 1963.

279 [26] J. Korringa, Physica 16(7) (1950) 601-610.

280 [27] W.W. Warren, Phys. Rev. B 3(11) (1971) 3708-3724.

281 [28] R. Dupree, D.J. Kirby, W. Freyland, Philos. Mag. B 46(6) (1982) 595-606.

282 [29] D. Turnbull, Solid State Physics, Elsevier1956, pp. 225-306.

283 [30] X. Liu, S.Y. Liu, E.Y. Chen, et al., J. Phys. Chem. Lett. 10(15) (2019) 4285-4290.

284 [31] C. Way, P. Wadhwa, R. Busch, Acta Mater. 55(9) (2007) 2977-2983.

285 [32] S. Wei, F. Yang, J. Bednarcik, et al., Nat. Commun. 4 (2013) 2083.

286 [33] M.E. Blodgett, T. Egami, Z. Nussinov, K.F. Kelton, Sci. Rep. 5(1) (2015) 13837.

287 [34] H. Tanaka, Phys. Rev. E 62(5) (2000) 6968-6976.

288 [35] H. Tanaka, Eur. Phys. J. E 35(10) (2012) 113.

289 [36] C.S. Ray, S.T. Reis, R.K. Brow, et al., J. Non-Cryst. Solids 351(16) (2005) 1350-

290

291

292

293

294

295

296

297

298

299 1358.

[37] Q.J. Zheng, Y.F. Zhang, M. Montazerian, et al., Chem. Rev. 119(13) (2019) 78487939.

[38] S. Lan, Y. Ren, X.Y. Wei, et al., Nat. Commun. 8 (2017) 14679.

[39] Q. Du, X.J. Liu, H.Y. Fan, et al., Mater. Today 34 (2020) 66-77.

[40] Z.P. Lu, C.T. Liu, Phys. Rev. Lett. 91(11) (2003) 115505.

[41] R. Grange, J. Kiefer, Trans. ASM 29(1) (1941) 85.

[42] S. Mukherjee, Z. Zhou, J. Schroers, et al., Appl. Phys. Lett. 84(24) (2004) 50105012.

300

[43] Y. Zhao, S. Kou, H. Suo, et al., Mater. Des. 31(2) (2010) 1029-1032. 

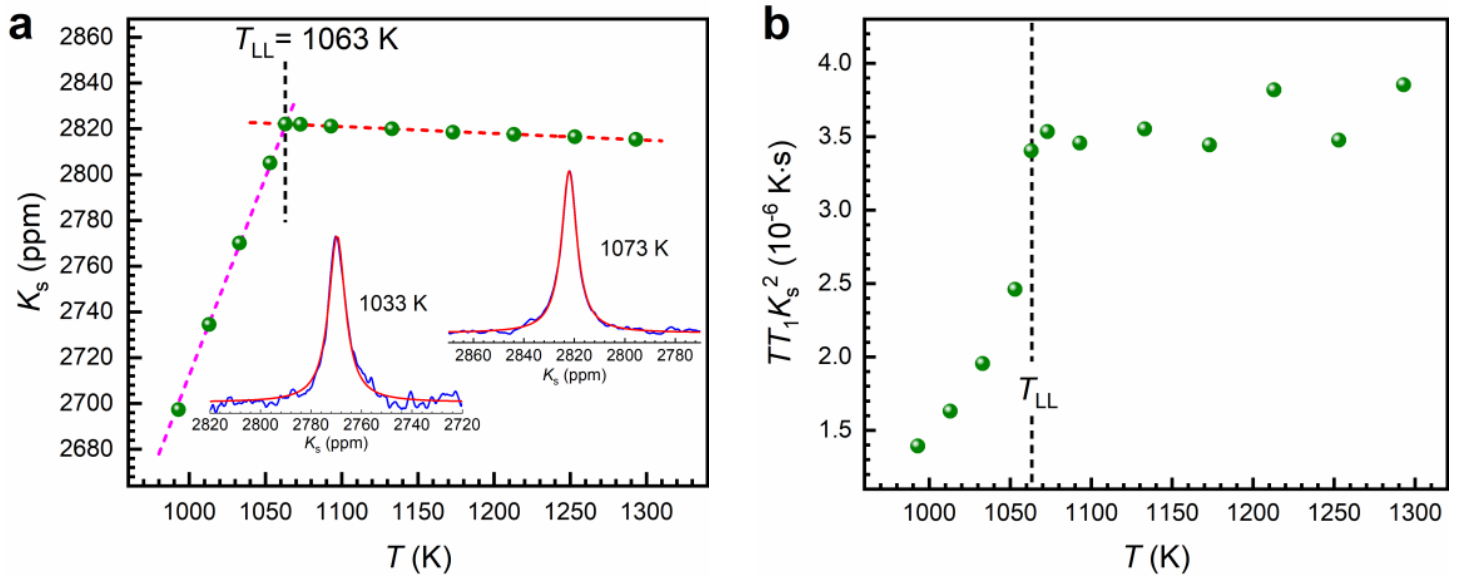

301
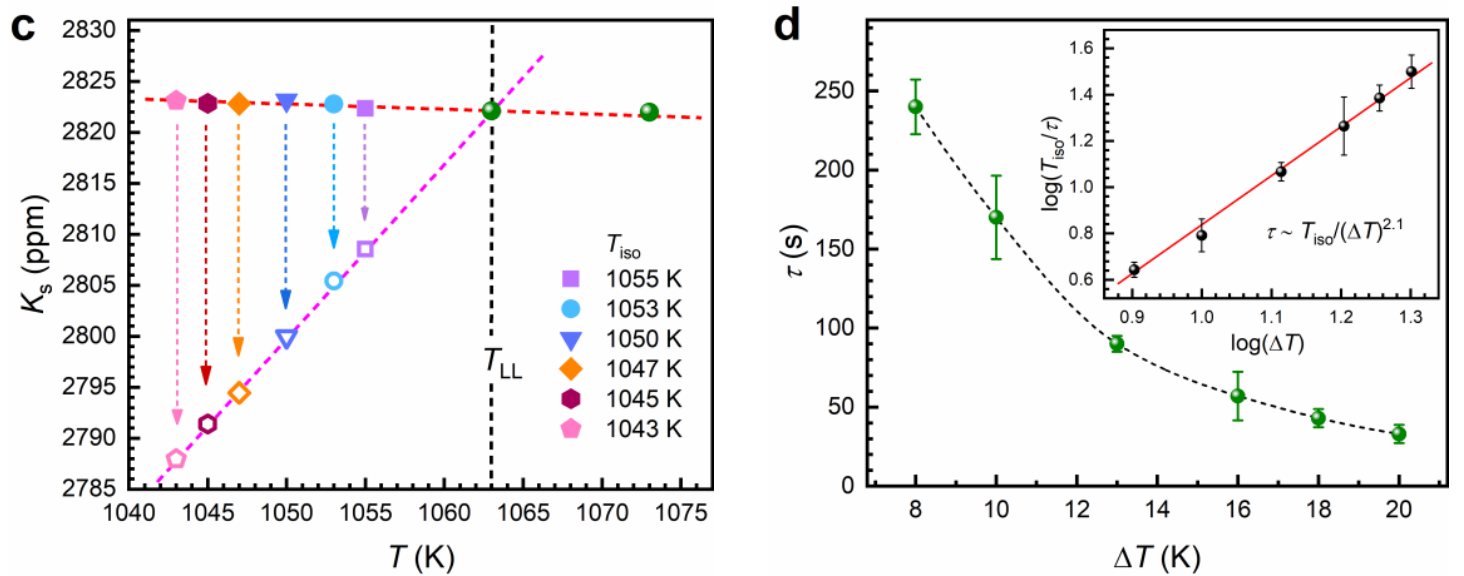

303 Fig. 1. Liquid-liquid transition in the equilibrium $\mathrm{Pd}_{42.5} \mathrm{Ni}_{42.5} \mathrm{P}_{15}$ melt characterized by

304 NMR. (a) Temperature dependence of ${ }^{31} \mathrm{P}$ Knight shift $\left(K_{\mathrm{s}}\right)$. The two linear fits (red and 305 pink dashed lines) intersect at $1063 \mathrm{~K}$. The insets are two typical spectra (blue line) above 306 and below the transition temperature with the Lorentzian fitting of the spectrum (red line).

307 (b) Value of $T T_{1} K_{\mathrm{s}}^{2}$ as a function of temperature. (c) The changes of $K_{\mathrm{s}}$ at different 308 undercooled temperatures $T_{\text {iso }}$ after quenching the melt from $1173 \mathrm{~K}$. The solid and open 309 symbols represent the initial and equilibrium $K_{\mathrm{s}}$, respectively. (d) The average incubation

310 time $\tau$ versus degree of undercooling $\Delta T=1063-T_{\text {iso. The inset plots } \log \left(T_{\text {iso }} / \tau\right) \text { versus }}$ $311 \log (\Delta T)$ and the slope of the linear fit is 2.1 . 

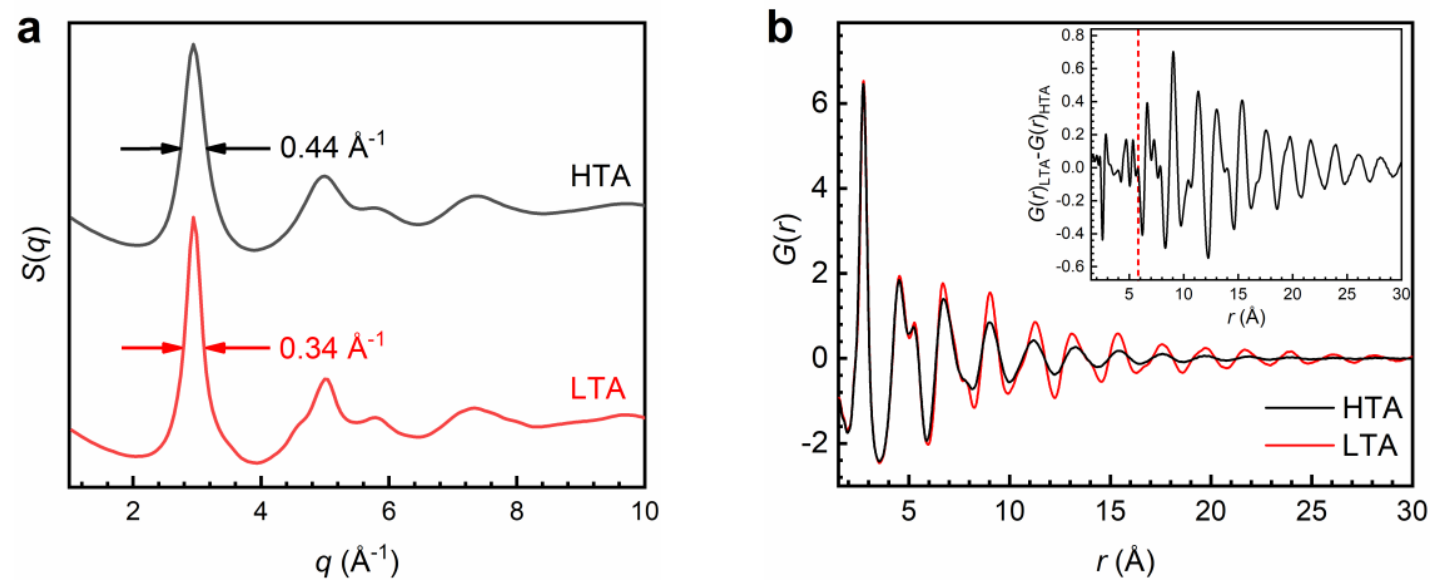

314 Fig. 2. Structural difference of HTA and LTA characterized by high-energy synchrotron 315 XRD. (a) Structure factors $S(q)$ of HTA and LTA. (b) Pair correlation functions $G(r)$ of 316 HTA and LTA. The inset shows the difference in $G(r)$ between LTA and HTA.
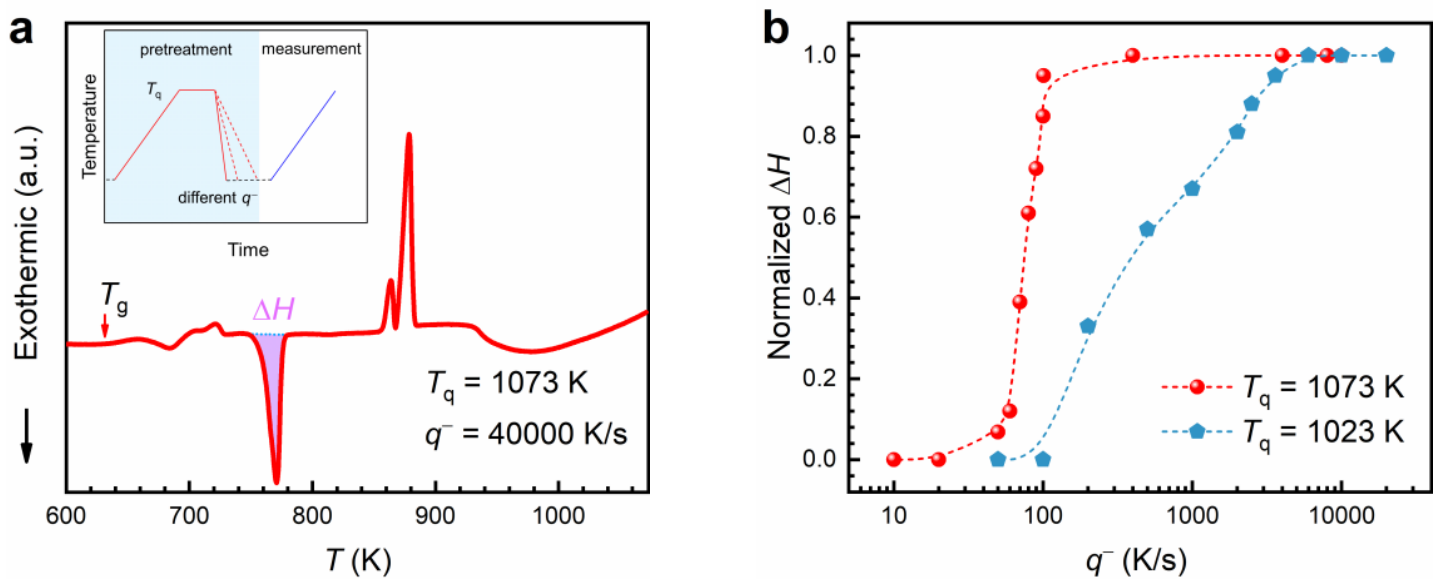

319 Fig. 3. Glass-forming ability of the two liquids characterized by FDSC. (a) A typical FDSC 320 heating curve of the sample obtained from $T_{\mathrm{q}}=1073 \mathrm{~K}$ and $q^{-}=40000 \mathrm{~K}$. The enthalpy of 321 crystallization is denoted as $\Delta H$. The inset shows the temperature protocol of the FDSC 322 experiments. (b) The content of amorphous phase as a function of cooling rate $q^{-}$of the 323 samples obtained from $T_{\mathrm{q}}=1073 \mathrm{~K}$ and $1023 \mathrm{~K}$, respectively. 

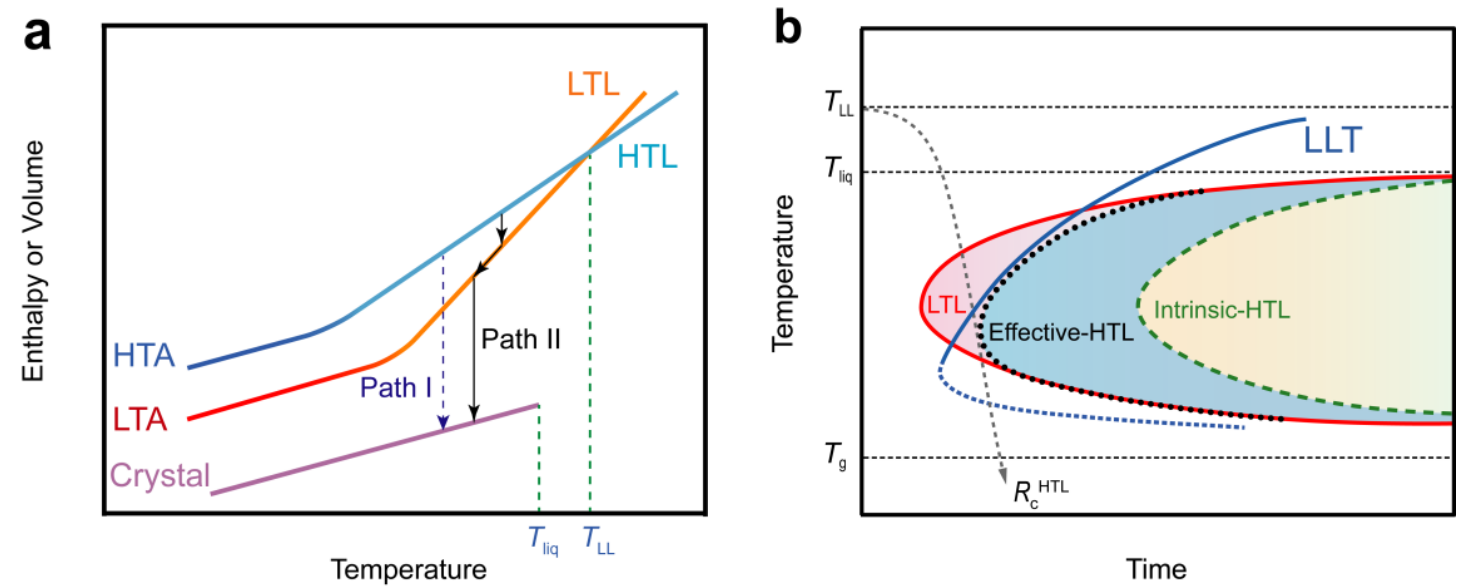

326 Fig. 4. Effects of LLT on the pathways and kinetics of crystallization. (a) Schematic 327 evolution of enthalpy or volume upon cooling the melt. (b) Mechanism of the different 328 GFA of HTL and LTL. The red and green dashed lines represent the intrinsic crystallization 329 TTT curve of LTL and HTL, respectively. The cerulean line represents the TTT curve of 330 LLT. The TTT curve of LLT intersects the TTT curve of LTL at high and low temperature 331 and the black dotted line denotes the effective crystallization TTT curve of HTL with its 332 nose around the low intersection. $R_{\mathrm{c}}^{\mathrm{HTL}}$ is the critical cooling rate to bypass the effective 333 TTT curve of HTL. 